\title{
Congenital right pulmonary artery to left atrial fistula: Elective video-thoracoscopic stapling
}

Pascal-Alexandre Thomas, MD, ${ }^{\mathrm{a}}$ Camille Soulatges, MD, ${ }^{\mathrm{b}}$ Caroline Ovaert, MD, ${ }^{\mathrm{b}}$ and Loïc Mace, MD, Marseille, France

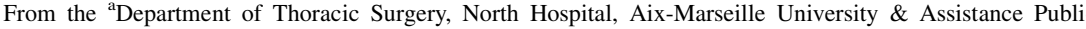

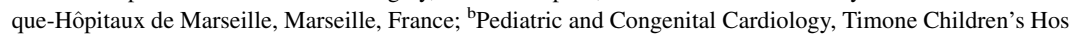
pital, Aix-Marseille University \& Assistance Publique-Hôpitaux de Marseille, Marseille, France; and ${ }^{\mathrm{c}}$ Thoracic and Cardiovascular Surgery, Timone Children's Hospital, Aix-Marseille University \& Assistance Publique-Hôpitaux de Marseille, Marseille, France.

Disclosures: Authors have nothing to disclose with regard to commercial support.

Received for publication Aug 23, 2017; revisions received Nov 5, 2017; accepted for publication Nov 28, 2017; available ahead of print Jan 6, 2018.

Address for reprints: Pascal-Alexandre Thomas, MD, Department of Thoracic Surgery, North Hospital, AixMarseille University \& Assistance Publique-Hôpitaux de Marseille, Chemin des Bourrely, 13915 Marseille,

France (E-mail: pathomas@ap-hm.fr).

J Thorac Cardiovasc Surg 2018; 156:e121-3

$0022-5223 / \$ 36.00$

Copyright (C) 2017 by The American Association for Thoracic Surgery

https://doi.org/10.1016/j.jtcvs.2017.11.094
}

Video clip is available online.

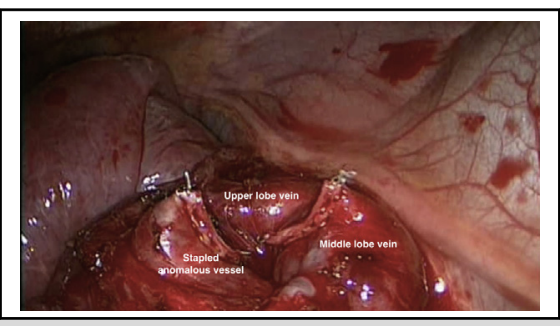

Stapled congenital right pulmonary artery-to-left atrial fistula via the right upper vein.

Central Message

Videothoracoscopic stapling is a safe and effective alternative to conventional open surgery or catheter closure for the treatment of congenital RPA to left atrial shunt.

See Editorial Commentaries pages e125 and e127.
Direct communication between the right pulmonary artery (RPA) and the left atrium (LA) is a rare congenital malformation of the pulmonary vasculature, but the true incidence remains unknown. It may result in chronic hypoxemia, cerebral systemic embolization or abscess, and congestive heart failure. In the reported case, transcatheter device closure was attempted but was considered hazardous. A complete cure was obtained with elective videothoracoscopic stapling of the fistula.

\section{CASE REPORT}

A 12-year-old boy, with normal physical development (164 cm/51 kg), was admitted to the school nursery because of seasonal gastroenteritis. The nurse noticed low $(89 \%)$ arterial oxygen saturation at routine pulse oxymetry and advised his parents to see a pediatrician. The child described dyspnea at exertion. On examination, there was no chest deformity, clubbing of the fingers, or murmur on chest auscultation. Subungual cyanosis was noticed. The arterial oxygen tension while in room air was $51 \mathrm{~mm} \mathrm{Hg}$, and carbon dioxide tension was $32.5 \mathrm{~mm} \mathrm{Hg}$. At hyperoxia test, breathing $100 \%$ oxygen for 20 minutes, the partial pressure of oxygen in the arterial blood only increased to $85 \mathrm{~mm} \mathrm{Hg}$, demonstrating a $30 \%$ shunting of the whole heart flow.
Transthoracic echocardiography showed normal-sized cardiac chambers and an intact interatrial septum. Contrast echocardiography revealed contrast bubbles in the LA within 4 cardiac cycles, suggesting the presence of an extra cardiac right-to-left shunt. Chest computed tomography scan showed normal lung 3-lobe anatomy and pulmonary veins pattern but a huge intraparenchymal pulmonary artery-to-left atrial fistula (Figure 1). Cardiac catheterization was performed to attempt device closure of the fistula, but the procedure was abandoned on the basis of location, size, and tortuous path of the aberrant vessel (Video 1).

A thoracoscopic surgical approach for elective ligation of the fistula in the left semilateral position was planned after informed consent, under general anesthesia with double-lumen-tube endobronchial intubation and 1-lung ventilation. The operation consisted of a right 3-port VATS posterior approach using a $30^{\circ}$ videothoracoscope. Ligation and division of the fistula were performed using a vascular stapler. The chest tube was removed at day 1 , and the patient was discharged on day 2 , with an oxygen saturation of $98 \%$ at room air. Postoperative follow-up at 6 months showed completed relief of symptoms and no surgery-related complications. Infused chest computed tomography scan showed normal pulmonary vessels anatomy. 


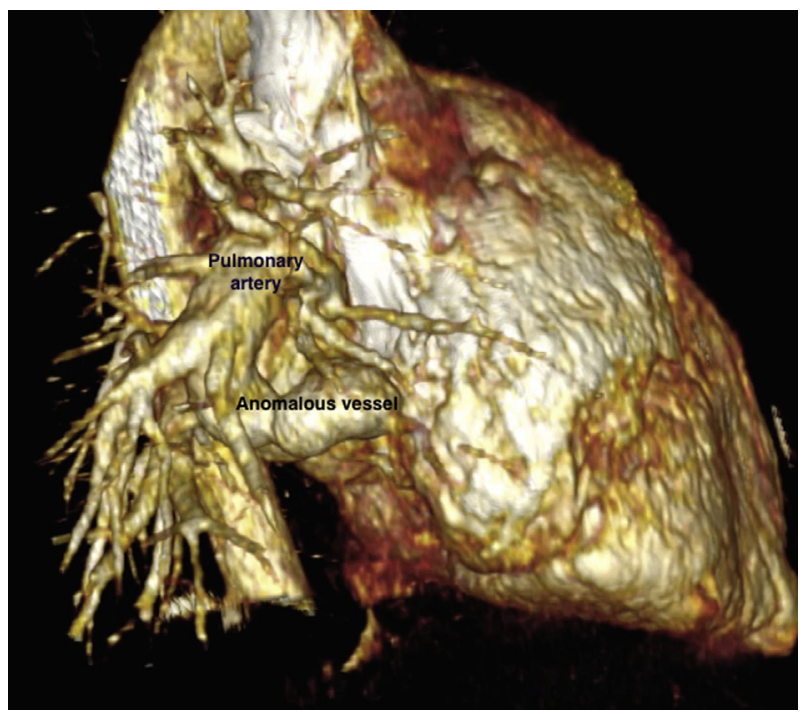

FIGURE 1. Chest computed tomography scan with 3-dimensional reconstruction (right lateral view) showing an RPA-to-LA fistula consisting of a large tortuous vessel originating from the posteroinferior aspect of the distal RPA and entering the LA at the level of the right upper pulmonary vein.

\section{DISCUSSION}

Friedlich and colleagues ${ }^{1}$ first described congenital direct communication between the RPA and the LA. In 2005, a review collecting 59 cases summarized the current knowledge on this intriguing rare entity described a 4-category classification of RPA to LA fistula (Appendix E1). ${ }^{2}$ Our case does not fit well into this classification. It can be linked to type 1 because the patient had a rather normal 3-lobe pulmonary anatomy and venous drainage pattern. However, the anomaly did not originate from the proximal RPA and did not join the LA posteriorly between the right and the left pulmonary veins, as typically reported. Indeed, the aberrant vessel originated more distally in the right pulmonary artery, as encountered in type 2 , but joined the LA laterally via the right upper vein, while receiving the venous drainage of the middle lobe. We deem that this particular pattern made any attempt of transcatheter occlusion hazardous. The antegrade deployment of the device from the right ventricle to the main pulmonary artery would have compromised the distal RPA flow. The veno-venous route from the femoral vein with a transseptal puncture would have carried the risk to impede the right upper pulmonary vein flow resulting in pulmonary edema and gangrene, at least of the middle lobe.

\section{CONCLUSIONS}

Even if several sporadic cases have been tackled by the transcatheter technique with various types of devices and

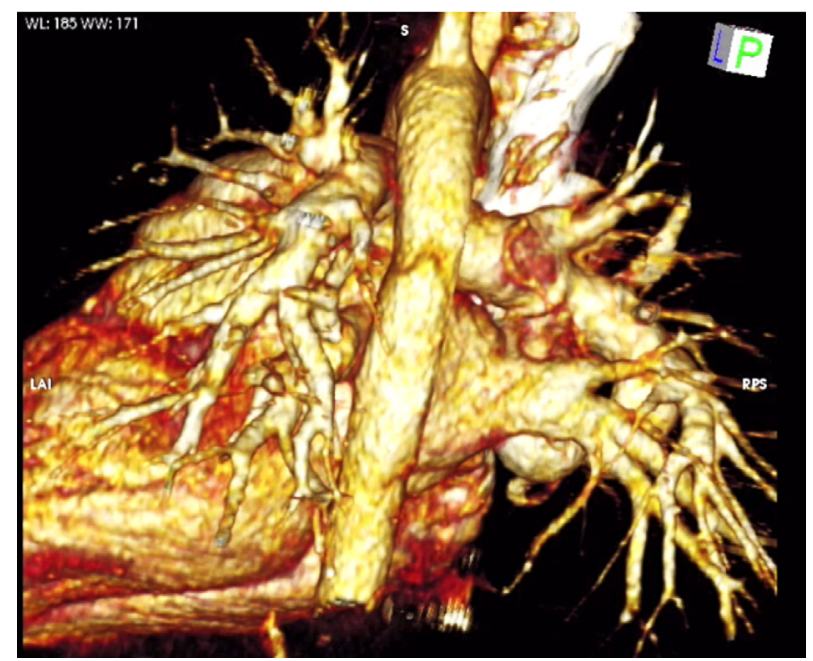

VIDEO 1. Preoperative imaging with 3-dimensional reconstructions and diagnostic angiography disclosing a tortuous intraparenchymal right pulmonary artery-to-left atrial fistula. Surgical exploration showing the abnormal vessel entering the LA separate from the upper root of the right upper pulmonary vein, but receiving the middle vein drainage before its extrapericardial termination. Dissection at the pulmonary hilum allowed the encirclement and stapling of the vessel upstream of the middle lobe vein drainage to avoid any obstruction to the pulmonary venous flow. Video available at: http://www.jtcvsonline.org/article/S0022-5223(17)32887-8/ fulltext.

coils since $2000,{ }^{3}$ elective surgery via thoracotomy or sternotomy remains the reference treatment of this condition. Three-dimensional chest computed tomography scan angiography was helpful to understand the pulmonary vessels anatomy ${ }^{4}$ and to plan the surgery. We favored a lateral approach over median sternotomy because the anomaly looked to be entirely located extrapericardically. We also aimed to avoid any sacrifice in lung parenchyma to preserve the pulmonary function. A video-thoracoscopic technique was successfully used to speed up the postoperative recovery. To the best of our knowledge, this strategy, which proved to be a safe and effective alternative to both conventional surgery and catheter closure, has not been reported.

\section{References}

1. Friedlich A, Bling RJ, Blount SG. Physiological studies in congenital heart disease. Circulatory dynamics in the anomalies of venous return to the heart including pulmonary arteriovenous fistula. Bull Johns Hopkins. 1950;86:20-57.

2. Chowdhury UK, Kothari SS, Airan B, Subramaniam KG, Venugopal P. Right pulmonary artery to left atrium communication. Ann Thorac Surg. 2005;80:365-70.

3. Slack MC, Jedeikin R, Jones JS. Transcatheter coil closure of a right pulmonary artery to left atrial fistula in an ill neonate. Catheter Cardiovasc Interv. 2000;50: 330-3.

4. Remy J, Remy-Jardin M, Artaud D, Fribourg M. Multiplanar and threedimensional reconstruction techniques in CT: impact on chest diseases. Eur Radiol. 1998;8:335-51. 


\section{APPENDIX E1. VARIOUS TYPES OF RIGHT} PULMONARY ARTERY TO LEFT ATRIUM COMMUNICATIONS

Type I: Anomalous vessel connecting the posterior aspect of the proximal RPA and draining into the LA with normal pulmonary venous connections.

Type II: Anomalous vessel connecting the lower lobe branch of the RPA and draining into the LA at the level of the right inferior pulmonary vein, which is absent. The vessel is aneurysmal proximal to its junction with the LA.

Type III: All pulmonary veins draining into the aneurysmal pouch between the RPA and the LA.

Type IV: Very proximal pulmonary arteriovenous fistula joining the LA. The right-sided pulmonary veins join the fistulous tract and the left-sided pulmonary veins join the LA directly. 